MATEC Web of Conferences 22,05015 (2015)

DOI: $10.1051 /$ matecconf/ 20152205015

(C) Owned by the authors, published by EDP Sciences, 2015

\title{
Simulation and Optimization of Strengthening the Gas-Liquid Mass Transfer Structure in Tray Column
}

\author{
Jihai Duan, He Hou, Huawei Sun \& Zisheng Zhang* \\ College of Chemical Engineering, Qingdao University of Science and Technology, Qingdao, Shandong, China
}

\begin{abstract}
This paper presented the analysis of the effect of the mesh layers, installation height and opening rate on gas-liquid two-phase flow field by numerical simulation, which based on Euler-Euler model and identified the best liquid-gas ratio. The simulation results showed that: when the installation height of the square mesh was $25 \mathrm{~mm}$ and opening rate was $55.7 \%$, the gas holdup distribution in the upper part of the mesh became higher and improved the liquid velocity distribution. Meanwhile, the gas-liquid distribution on trays was more uniform.
\end{abstract}

Keywords: Euler-Euler model; liquid-gas ratio; gas-liquid distribution

\section{INTRODUCTION}

Tray Column is considered as one of the most important gas-liquid and liquid-liquid mass transfer equipments. Because of its simple structure, easy amplification and low cost, it is always gaining much attention [1-2]. The hydrodynamic characteristics of the gas-liquid two-phase on the tray column affect the chemical reaction and the efficiency of the tray [3]. So a lot of tray flow field measurements had been carried out at home and abroad. However, the gas-liquid two-phase flow on the tray is a particular form and the two-phase interaction is very complex, which makes the experimental work difficulty and the cost is very high.

Compared with the traditional methods of chemical engineering experiment, numerical simulation costs less, takes a shorter time, and it also can be more convenient to change operating conditions according to the different actual needs [4]. The essential difference between CFD and other technologies is that it is based on the physical analysis, rather than mathematical deduction, which is more close to reality. In the process of numerical simulation, CFD can deepen the understanding of the physical process, which is very useful in the analysis and interpretative in the results [5-7].In order to overcome the problem of trace distillation, where recombinant concentration is very low whereas the demand of separation remains is high, $\mathrm{Li}$ Suya[8] proposed a method by adding mesh on the ordinary sieve tray to achieve the separation effect, and this method was proved effectively by experiments. Based on Li's study, by using of large commercial CFD fluid simulation software FLUENT 6.3.26, this paper aims to find out the best configuration condition through analyzing the effect of the mesh layers, installation height, and opening rate on gas-liquid two-phase flow field[8].

\section{PHYSICAL AND MATHEMATICAL MODEL}

\subsection{Physical model}

The simulation object is gas-liquid phase on sieve trays at $1 \mathrm{~atm}$ under the air-water system. For a plane which parallels the tray, the fluid-flow is considered to be the center symmetric flow, so the computational area is defined as half block tray section along the center line of symmetry, which can save calculation time. Compared with the diameter of the tray, the thickness of the tray is so small that it can be neglected. In this paper, it assumes that the tray wall has no thickness. In this case, it is possible for the tray to generate the high quality structure grid .The calculation area is shown in Figure 1.

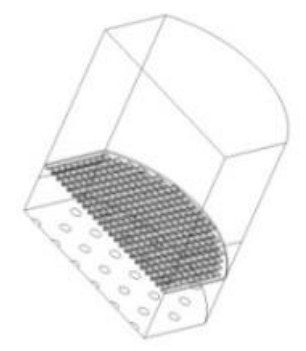

Figure 1. Physical model of distillation column

Two mesh shapes are simulated in this paper, which are square mesh and circular mesh. The size of square mesh is $3 \mathrm{~mm} \times 3 \mathrm{~mm}$, interval is $1 \mathrm{~mm}$, the opening rate is $55.7 \%$, the diameter of circular mesh is $3 \mathrm{~mm}$, the center distance is $4 \mathrm{~mm}$ and the opening rate is $51.4 \%$.

*Corresponding author: ZZZSLunWen@,126.com

This is an Open Access article distributed under the terms of the Creative Commons Attribution License 4.0, which permits unrestricted use, distribution, and reproduction in any medium, provided the original work is properly cited. 


\subsection{Mathematical model}

According to the basic idea of two-phase flow model [9], gas-liquid two-phase flow on the tray are considered as continuous phase respectively. Each phase has its own properties of fluid. The contact of two phase is achieved by mutual coupling [10].Considering the relationship between the phase interface, first we set up the local and instantaneous conservation equations for each phase, and then get the expression of two-phase flow equations and various interaction between phases by the Reynolds average method. In this way, the control equations of the two-phase flow can be deduced. Furthermore, the classical k- $\varepsilon$ double equation model was used for turbulence closure [11-14]. The control equations include the following components:

1) The mass conservation equation:

$\frac{\partial\left(r_{\alpha} \rho_{\alpha} u_{\alpha}\right)}{\partial x}+\frac{\partial\left(r_{\alpha} \rho_{\alpha} v_{\alpha}\right)}{\partial y}+\frac{\partial\left(r_{\alpha} \rho_{\alpha} w_{\alpha}\right)}{\partial z}=0$

2) The momentum equation:

$u_{\alpha} \frac{\partial\left(r_{\alpha} \rho_{\alpha} u_{\alpha}\right)}{\partial x}+v_{\alpha} \frac{\partial\left(r_{\alpha} \rho_{\alpha} u_{\alpha}\right)}{\partial y}+w_{\alpha} \frac{\partial\left(r_{\alpha} \rho_{\alpha} u_{\alpha}\right)}{\partial z}=-r_{\alpha} \frac{\partial p_{\alpha}}{\partial x}$

$+\frac{\partial}{\partial x}\left(r_{\alpha} u_{\alpha} \frac{\partial u_{\alpha}}{\partial x}\right)+\frac{\partial}{\partial y}\left(r_{\alpha} u_{\alpha} \frac{\partial u_{\alpha}}{\partial y}\right)+\frac{\partial}{\partial z}\left(r_{\alpha} u_{\alpha} \frac{\partial u_{\alpha}}{\partial z}\right)+M_{\alpha, u}$

$u_{\alpha} \frac{\partial\left(r_{\alpha} \rho_{\alpha} v_{\alpha}\right)}{\partial x}+v_{\alpha} \frac{\partial\left(r_{\alpha} \rho_{\alpha} v_{\alpha}\right)}{\partial y}+w_{\alpha} \frac{\partial\left(r_{\alpha} \rho_{\alpha} v_{\alpha}\right)}{\partial z}=-r_{\alpha} \frac{\partial p_{\alpha}}{\partial x}$

$+\frac{\partial}{\partial x}\left(r_{\alpha} u_{\alpha} \frac{\partial u_{\alpha}}{\partial x}\right)+\frac{\partial}{\partial y}\left(r_{\alpha} u_{\alpha} \frac{\partial u_{\alpha}}{\partial y}\right)+\frac{\partial}{\partial z}\left(r_{\alpha} u_{\alpha} \frac{\partial u_{\alpha}}{\partial z}\right)+M_{\alpha, v}$

$u_{\alpha} \frac{\partial\left(r_{\alpha} \rho_{\alpha} w_{\alpha}\right)}{\partial x}+v_{\alpha} \frac{\partial\left(r_{\alpha} \rho_{\alpha} w_{\alpha}\right)}{\partial y}+w_{\alpha} \frac{\partial\left(r_{\alpha} \rho_{\alpha} w_{\alpha}\right)}{\partial z}=-r_{\alpha} \frac{\partial p_{\alpha}}{\partial x}$

$+\frac{\partial}{\partial x}\left(r_{\alpha} u_{\alpha} \frac{\partial u_{\alpha}}{\partial x}\right)+\frac{\partial}{\partial y}\left(r_{\alpha} u_{\alpha} \frac{\partial u_{\alpha}}{\partial y}\right)+\frac{\partial}{\partial z}\left(r_{\alpha} u_{\alpha} \frac{\partial u_{\alpha}}{\partial z}\right)+M_{\alpha, w}$

Where $a=L / G, M_{a}$ is the interphase force [12]. In the model, the drag force and virtual mass force were considered. The drag force:

$\vec{F}_{v m}=0.5 \alpha_{p} \rho_{q}\left(\frac{d_{q} \vec{v}_{q}}{d t}-\frac{d_{p} \vec{v}_{p}}{d t}\right)$

$\frac{d_{p}(\phi)}{d t}=\frac{\partial(\phi)}{\partial t}+\left(\vec{v}_{q} \cdot \vec{v}\right) \phi$

The virtual mass force [13] used Schiller and Naumann formula[14]

$$
\begin{aligned}
f & =\frac{C_{D} \mathrm{Re}}{24} \\
C_{D} & =\left\{\begin{array}{l}
24\left(1+0.15 \mathrm{Re}^{0.687}\right) / \mathrm{Re} \\
0.44
\end{array}\right.
\end{aligned}
$$

Where ${ }^{\mu_{\alpha}}$ and ${ }^{\mu_{t a}}$ are effective eddy viscosity coefficients, and $\mu_{\alpha}=\mu_{t a}+\mu_{m \alpha} . \mu_{m \alpha}$ is a molecular viscosity coefficient.

3) Conservation equation of volume:
$\sum_{a=1}^{N_{p}} r_{a}=1$

4) The gas phase and liquid phase shared the same pressure field:

$p_{a}=p$

5) Liquid phase turbulence model used mixed phase $\mathrm{k}-\varepsilon$ turbulence model [15], equation is as follows:

$u_{a} \frac{\partial\left(r_{a} \rho_{a} k_{a}\right)}{\partial x}+v_{a} \frac{\partial\left(r_{a} \rho_{a} k_{a}\right)}{\partial y}+w_{a} \frac{\partial\left(r_{a} \rho_{a} k_{a}\right)}{\partial z}=\frac{\partial}{\partial x}\left(\left(u+\frac{u_{t a}}{\sigma_{k}}\right) \frac{\partial k_{a}}{\partial x}\right.$

$+\frac{\partial}{\partial y}\left(\left(u+\frac{u_{t a}}{\sigma_{k}}\right) \frac{\partial k_{a}}{\partial y}\right)+\frac{\partial}{\partial z}\left(\left(u+\frac{u_{t a}}{\sigma_{k}}\right) \frac{\partial k_{a}}{\partial z}\right)+r_{a}\left(p_{a}-p_{a} \varepsilon_{a}\right)$

$u_{a} \frac{\partial\left(r_{a} p_{a} \varepsilon_{a}\right)}{\partial x}+v_{a} \frac{\partial\left(r_{a} p_{a} \varepsilon_{a}\right)}{\partial y}+w_{a} \frac{\partial\left(r_{a} p_{a} \varepsilon_{a}\right)}{\partial z}=\frac{\partial}{\partial x}\left(\left(u+\frac{u_{t a}}{\sigma_{k}}\right) \frac{\partial \varepsilon_{a}}{\partial x}\right)$

$\left.+\frac{\partial}{\partial y}(1)\left(u+\frac{u_{t a}}{\sigma_{k}}\right) \frac{\partial \varepsilon_{a}}{\partial y}\right)+\frac{\partial}{\partial z}\left(\left(u+\frac{u_{t a}}{\sigma_{k}}\right) \frac{\partial \varepsilon_{a}}{\partial z}\right)+r_{a} \frac{\varepsilon_{a}}{k_{a}}\left(c_{s 1} p_{a}-c_{s 2} p_{a} \varepsilon_{a}\right)$

$p_{a}=u_{t}\left\{2\left(\left[\left(\frac{\partial u_{a}}{\partial x}\right)^{2}+\left(\frac{\partial v_{a}}{\partial y}\right)^{2}+\left(\frac{\partial w_{a}}{\partial z}\right)^{2}\right]\right)+\left(\frac{\partial v_{a}}{\partial x}+\frac{\partial u_{a}}{\partial y}\right)^{2}\right.$

$\left.+\left(\frac{\partial w_{a}}{\partial y}+\frac{\partial v_{a}}{\partial z}\right)^{2}+\left(\frac{\partial u_{a}}{\partial z}+\frac{\partial w_{a}}{\partial x}\right)^{2}\right)$

$\mu_{t L}=C_{\mu} \rho_{\alpha} \frac{k_{L}^{2}}{\varepsilon_{L}}+\mu_{t p}$

Using the zero equation turbulence model, the contribution of gas relative turbulence selected Sato Turbulence Enhancement model [16]:

$\mu \mathrm{tp}=\mathrm{C} \mu \sigma \mathrm{LrGdG}|\mathrm{UG}-\mathrm{UL}|$

Based on the two fluid Eulerian two-phase flow model, with the aqueous phase as the main phase, the air as the second phase, this paper established a mathematical model of gas-liquid two-phase of sieve tray, which took the influence of the virtual mass force and drag force into account. Due to the unclosed of the equation, the turbulent model was needed [17].

\subsection{Boundary condition}

(1)The inlet boundary

It is assumed that the inlet velocity has a uniform distribution:

$u_{\text {Lin }}=u_{0}$

$v_{\text {Lin }}=u_{0}$

$w_{\text {Lin }}=u_{0}$

For the inlet boundary using the turbulence degree and the hydraulic diameter [18]:

$I=0.16\left(\operatorname{Re}_{D_{H}}\right)^{-1 / 8}$ 
$\mathrm{D}_{\mathrm{H}}=\frac{4 \times \text { Cross sectional area }}{\text { Infiltration of the surrounding }}$

Gas phase: when the gas phase goes into the tray through the sieve pores, it assumes that the flow velocity is uniformly distributed in the hole entrance, by satisfying gas phase continuity [19].

$u_{\text {Gin }}=0$

$v_{\text {Gin }}=0$

$w_{\text {Gin }}=\frac{u_{s}}{\text { Opening rate }}$

According to the method above: the liquid phase hydraulic diameter $\mathrm{DH} 1=43.18 \mathrm{~mm}$; the gas phase hydraulic diameter $\mathrm{DH} 2=5 \mathrm{~mm}$; the liquid phase turbulence intensity $\mathrm{I} 1=5.6227 \%$; the gas phase turbulence intensity $\mathrm{I} 2=6.7922 \%$; the inlet velocity of liquid phase $\mathrm{uLin}=0.1 \mathrm{~m} / \mathrm{s}$; and the inlet velocity of gas phase wGin $=2.78 \mathrm{~m} / \mathrm{s}$.

\section{(2)Outlet boundary}

At the top of the gas phase and liquid phase on the weir, we set the boundary condition of pressure and relatively gauge to zero, then:

$$
P=0
$$

(3)Boundary conditions on the outlet weir of the wall surface and the floor

For the wall surface, the floor and the outlet weir near wall, the velocity gradient is large, so the boundary takes the wall- function method. It assumed that the gas phase and liquid phase relative to the wall had no slip motion and Nallasamy logarithmic wall law method was used for the liquid phase [20-21].

For the tray wall:

$u_{L}=\frac{u_{\tau}}{k} \ln \left(y^{+} E\right)$

$v_{L}=0$

$w_{L}=\frac{u_{\tau}}{k} \ln \left(y^{+} E\right)$

For the floor:

$u_{L}=\frac{u_{\tau}}{k} \ln \left(z^{+} E\right)$

$v_{L}=\frac{u_{\tau}}{k} \ln \left(z^{+} E\right)$

$w_{L}=0$

This paper simulated the flow of the non steady state of the whole micro plate in the 30 s time by Flu- ent6.3.26. Since the error of model was smaller at higher superficial gas velocity and the calculation can be convergent after $5 \mathrm{~s}$, this paper selected $\mathrm{t}=25 \mathrm{~s}$, and superficial gas velocity was $2.78 \mathrm{~m} / \mathrm{s}$ to study tray gas-liquid flow field and carried on the contrast analysis.

\section{INFLUENCES OF SIEVE MESH INSTALLA- TION HEIGHT}

\subsection{Effect on gas holdup}

The distribution of gas holdup at $y=10 \mathrm{~mm}$ with the different installation height of sieve mesh is shown in Figure 2. When the installation height is $25 \mathrm{~mm}$, the gas holdup becomes the highest and its distribution is the most uniform. The reason for this phenomenon is that this height can be better to break the big bubble, make the bubble diameter smaller and gas phase distribution more uniform. Furthermore, in the liquid entrance, gas holdup is particularly low, whereas in the relative position, it is high. The reason for this case is that gas coning leads to uneven distribution of gas-liquid phase, and some local vortex core are formed which makes the gas-liquid mass transfer not uniform. If the sieve mesh is arranged higher or lower, the breaking effects on the local vortex core are not obvious and the effect of gas-liquid mass transfer is

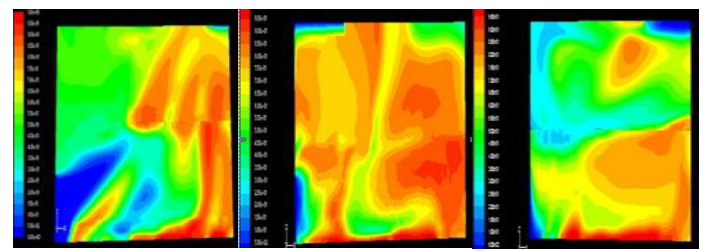

Figure 2. Distribution of gas hold up at $\mathrm{y}=10 \mathrm{~mm}(\mathrm{H}=20,25$, $30 \mathrm{~mm})$

not ideal.

The distribution of gas holdup at $\mathrm{z}=22,27,32 \mathrm{~mm}$ with the different installation heights of sieve mesh is shown in Figure 3. When the installation height is 25 $\mathrm{mm}$, the gas holdup becomes the highest and its distribution is the most uniform. It shows that the existence of mesh break big bubbles into smaller ones, which makes the gas holdup increases. When the installation height is $20 \mathrm{~mm}$ or $30 \mathrm{~mm}$, the sieve mesh also plays a certain role, but the effect is not obvious and boundaries still exist obviously between gas-liquid phases. 


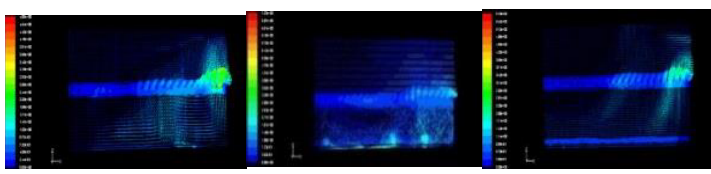

Figure 4. Distribution of liquid velocity at $\mathrm{y}=10 \mathrm{~mm}(\mathrm{H}=20,25$, $30 \mathrm{~mm}$ )

\subsection{Effect on liquid velocity}

The distribution of liquid velocity at $\mathrm{y}=20 \mathrm{~mm}$ with the different installation height of sieve mesh is shown in figure 4. When the installation height is $25 \mathrm{~mm}$, the distribution of liquid velocity can be improved. Since at the beginning of the gas phase velocity is larger, gas coning and local vortex are formed, which will cause uneven distribution of liquid phase and the gas-liquid mass transfer is impeded. When the liquid phase continues to rise, it meets sieve mesh, and then the gas coning is broken, the local eddy is dispersed. The liquid velocity distribution becomes uniform and the gas-liquid mass transfer is improved. Furthermore, it shows that at the exit of fluid, the distribution of velocity is not uniform, so that the velocity becomes large, which is unfavorable for mass transfer.

\section{INFLUENCE OF OPENING RATE}

\subsection{Effect on gas holdup}

The distribution of gas holdup at $\mathrm{y}=10 \mathrm{~mm}$ with circular mesh (opening rate is $51.4 \%$ and installation height is $25 \mathrm{~mm}$ ) is shown in Figure 5. Compared with square sieve (opening rate is $55.7 \%$ and installation height is $25 \mathrm{~mm}$ ) introduced in Figure 2 and Figure 3, the result shows that when the opening rate is lower in circular mesh, the gas holdup is lower too. However, its distribution is more uniform. Furthermore, the gas holdup distribution is not affected when adding the number of mesh and the effect of gas-liquid mass transfer is not obvious.

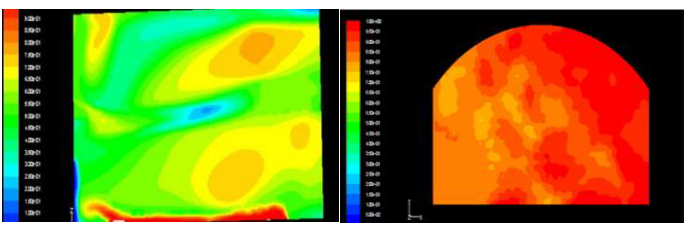

Figure 5. Distribution of gas volume fraction at $Y=10 \mathrm{~mm}$, $\mathrm{Z}=25 \mathrm{~mm}$

\subsection{Effect on liquid velocity}

The distribution of liquid velocity at $\mathrm{y}=10 \mathrm{~mm}$ with circular mesh (opening rate is $51.4 \%$ and installation height is $25 \mathrm{~mm}$ ) is shown in Figure 6. Compared with square sieve (opening rate is $55.7 \%$ and installation height is $25 \mathrm{~mm}$ ) introduced in Figure 7, the results show that adding a circular sieve also has an impact on weakening the effect of gas cone vortex on gas-liquid phase flow. However, the speed of the lower part or the higher part of the mesh is not uniform and the large vortex still exists, which is unfavorable for mass transfer. So the square sieve can play a better role of gas-liquid distribution, which is more conducive to the gas-liquid mass transfer and can improve

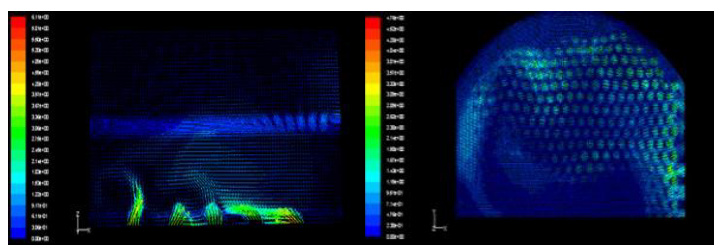

Figure 6. Distribution of water velocity at $y=10 \mathrm{~mm}, z=25 \mathrm{~mm}$ (cyclo-mesh)

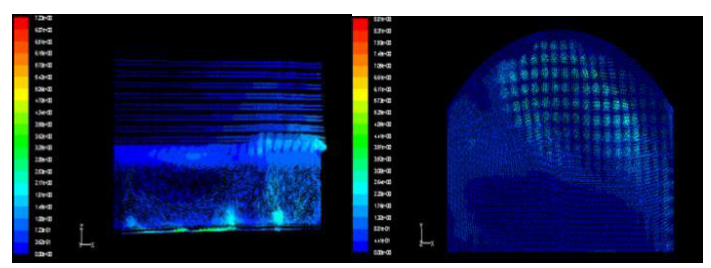

Figure 7. Stribution of water velocity at $y=10 \mathrm{~mm}, \mathrm{z}=25 \mathrm{~mm}$ the separation efficiency of the tray.

\section{CONCLUSIONS}

The whole micro tray set a single-layer mesh. When installation height of the square mesh was $25 \mathrm{~mm}$, the opening rate was $55.7 \%$, and the mesh was better able to break the bubbles, strengthen the surface renewal, and increase the contact area and the gas holdup, which on the upper part of mesh, leaded the distribution become more uniform. Simultaneously, mesh also had an impact on weakening the effect of gas cone vortex on gas-liquid phase flow, improved the distribution of liquid phase velocity and made the gas-liquid fluid better for mass transfer.

\section{ACKNOWLEDGMENTS}

This work is supported by National Natural Science

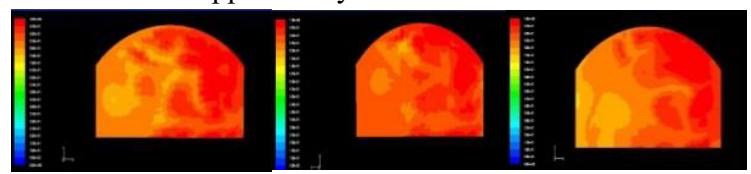

Figure 3. Distribution of gas holdup at $\mathrm{z}=22,27,32 \mathrm{~mm}(\mathrm{H}=20,25$ $30 \mathrm{~mm})$ 
Foundation of China (No.21276132) and Livelihood Project Foundation of Qingdao (13-1-3-128-nsh).

\section{REFERENCES}

[1] Li, C. L., Wang, Z.Y. \& Li, B. C., et al.2002.Latest Progress and Research Prospect of Tray. Journal of Hebei University of Technology. 31(1): 22.

[2] Wang, S. F. \& Xiang, S. G. 2014. Application and research progress of valve trays. Chemical Industry and Engineering Progress. 33(7): 1677-1696.

[3] Liu, D. X. 2008. CFD Modeling of Hydraulics and Mass Transfer of Distillation Trays and Experimental Studies of New Tray. Tianjin: Tianjin University.

[4] Zhang, P., Li, Y. X. \& Qin, R., et al. 2014. Three-Dimension Numeric Simulation of Liquid Flow on Oblique Sieve Tray. Journal of Chemical Engineering of Chinese Universities. 28(6): 1217-1222.

[5] Wang, X. L.2002.Three-dimensional fluid field and mass transfer simulation on distillation trays. China: Tianjin University.

[6] Krishna, R. 1999.CFD simulations of sieve tray hydrodynamics. Chem. Engng. Res. Design, Trans. I. Chem. E. 77: 639-646.

[7] Gao, C.H., Jiang, K. \& Zheng, S.Z, et al. 2012. CFD simulation of liquid flow field on double-pass overflow combined trapezoid spray tray. Chemical Engineering (China). 40(4):62-66.

[8] Guo, Y. F., Liu, H. \& Chen, B. H., et al. 2004. Numerical computation for turbulent flow of two phases on a sieve tray. Journal of Beijing University of Chemical Technology. 1(1): 2 .

[9] Wang, W.W., Li, S.Y., \&Li, J.L. 2012. Experimental Determination of Bubble Size Distributions in Laboratory Scale Sieve Tray with Mesh. Industrial \& Engineering Chemistry Research.51 (20): 7067-7072.

[10] Sundar, R. \& Tan, R. B. 1999.A model for bubble-to-jet transition at a submerged orifice. Chem. Eng. Sci. 18(54):4053-4060.

[11]Zhang, M. Q. \&Yu, G. C. 1994.Simulation of two dimensional liquid phase flow on a distillation tray. Chinese J. ChemEng. 2(2): 63.

[12] Calderbank, P. H. \& Rennie, J. 1962. The physical properties of foams and froths formed on sieve plates. Trans. Instn. Chem. Engrs. 40(1): 3-12.

[13] Hofhuis,P.A. \& Zuiderweg, F.J.1979. Sieve plates: dispersion density and flow regimes. Instn. Chem. Engr. Symp. Ser. 2(56): 21-26.

[14]Yakhot, V. \& Orzag, S. A. 1986.Renormalization group analysis of turbulence. I . basic theory. Journal of Scientific Computing. 1(1): 3-51.

[15]Locker, M. J.\& Kirkpatrick, R. D.1979. Uddin M S..Froth regime point efficiency for gas-film controlled mass transfer on a two-dimensional sieve tray. Trans. Inst. Chem Eng. 57:25-30.

[16] aten, J. M. 2000. Modeling sieve tray hydraulics using computational fluid dynamics. Chem. Eng. Jour. 77(3): 143-151.
[17] Gautreaux, M.F. \& O'Connell H.E.1955. Effect of length of liquid path on plate efficiency. Chem. Eng. Prog. 51(5): 232-237.

[18]Yu, G. Z. \& Gu, F. Z.1981. Simulation and efficiency of large tray (II)-Two dimensional fixed number. Journal of Chemical Industry and Engineering (China). 32(2):97-109.

[19] GetyeGesit, N. K. \& Chuang, K. T. 2003. CFD Modeling of flow patterns and Hydraulics of commercial-scale sieve tray. AIChE J. 49(4): 910-925.

[20] Li, L. C., Wang, J. J. \& Gu, X. P., et al. 2008.CFD simulation of gas holdup and gas-liquid flow in bubble columns. Science paper Online. 3(12): 885-889.

[21]Colbum, A. P.1936.Effect of entrainment on plate efficiency in distillation. Industrial \& Engineering Chemistry. 8(5): 526-544.

[22]Liu, M. S., Yang, F. \& Dong, Q. W., et al. 2010.Study of the Influence of Wall Surface Functions in Simulating a Fluid Laterally Sweeping a Tube Bundle. Journal of Engineering for Thermal Energy and Power. 25(5): 497-501. 